\title{
ID 3 Evolution of Structure in the Universe
}

\author{
Simon D. M. White \\ Max-Planck-Institut für Astrophysik, Garching-bei-München, 85748 Germany \\ email: swhite@mpa-garching.mpg.de
}

\begin{abstract}
Recent studies of the Cosmic Microwave Background have provided us with a high quality image of the Universe when it was only 380,000 years old. At that time it was a nearuniform mixture of hydrogen, helium, dark matter and radiation, with no galaxies, no stars, no planets and no people, indeed no atomic nuclei heavier than Lithium. Under the action of gravity, the weak fluctuations observed in the microwave sky evolved into the extraordinarliy complex structure of our present Universe. I will show how supercomputer simulations can be used to demonstrate that such evolution does indeed reproduce the observed properties of today's galaxies and large-scale structures, thus confirming the extraordinary assumptions of the current structure formation paradigm. Only a quarter of the energy density of the present Universe is in gravitating matter; only a sixth of this matter is made of atoms or other known particles; only 5 percent of this baryonic material is currently inside galaxies. Most of today's Universe is in the form of Dark Energy; most of the gravitating matter is Dark Matter; and most of the baryons remain unseen in intergalactic space. The properties of the fluctuations measured in the microwave sky suggest that they originated very close to the Big Bang as quantum fluctuations of the vacuum itself. Everything has formed from nothing.
\end{abstract}

Keywords. cosmic microwave background, dark matter, dark energy, evolution, gravity, galaxies, simulations, structure of Universe

Text of presentation not available. 